\title{
Genetic and structural identification of an O-acyltransferase gene (oacC) responsible for the 3/4-O-acetylation on rhamnose III in Shigella flexneri serotype 6
}

Yuriy A Knirel ${ }^{1 \dagger}$, Jianping Wang ${ }^{2 \dagger}$, Xia Luo ${ }^{2+}$, Sofya N Senchenkova ${ }^{1 \dagger}$, Ruiting Lan ${ }^{3+}$, Anna M Shpirt ${ }^{1}$, Pengcheng Dư ${ }^{2}$, Alexander S Shashkov ${ }^{1}$ Nan Zhang ${ }^{2}$, Jianguo Xu ${ }^{2}$ and Qiangzheng Sun ${ }^{2^{*}}$

\begin{abstract}
Background: O-antigen (O-polysaccharide) of the lipopolysaccharide is a highly variable cell component of the outer membrane in Shigella flexneri. It defines the serospecificity and plays an important role in the pathogenesis of shigellosis. There are two distinct O-antigen forms for the 19 serotypes of $S$. flexneri: one for serotypes 1-5, X, Y, 7 (and their subtypes), and the other for serotype 6. Although having different basal O-polysaccharide structures, the two forms share a common disaccharide fragment $\left.[\rightarrow 2)-a-L-R h a p^{\prime \prime \prime}-(1 \rightarrow 2)-a-L-R h a p^{\prime \prime}\right]$. In serotype 6 and some non-6 serotypes, Rha'"I is O-acetylated at position either 3 or 4 (3/4-O-acetylation), conferring to the hosts a novel antigenic determinant named O-factor 9 . An acyltransferase gene (oacB) responsible for this modification has been identified in serotypes 1a, 1b, 2a, 5a, and Y, but not in serotype 6 .

Results: Using genetic, serological, and chemical approaches, another acyltransferase gene named oacC was demonstrated to be responsible for the 3/4-O-acetylation on Rha"l in the O-antigen of S. flexneri serotype 6. Inactivation of the oacC gene resulted in the loss of the 3/4-O-acetyltion, and the cloned oacC gene restored this modification upon transformation. In accordance with the similarity in the acceptor substrate structure and high sequence homology (72\% identity) between oacC and $\mathrm{OacB}$, oacC has the interchangeable function with the oacB gene in mediation of the 3/4-O-acetylation. The oacC gene is located in a prophage on the chromosome and presented in all 77 serotype 6 strains tested.
\end{abstract}

Conclusions: Identification and functional characterization of the O-acetyltransferase encoding gene, oacC, shows that it is involved in O-antigen modification by 3/4-O-acetylation on Rha'l' specific to serotype 6.

Keywords: Shigella flexneri, 3/4-O-acetylation, Acyltransferase, oacC, O-antigen, Anti-O-factor 9 serum

\section{Background}

Shigella flexneri is the major pathogen causing bacillary dysentery (shigellosis) in developing countries. It is estimated that there are 125 million shigellosis cases annually in Asia, resulting in 14,000 deaths, the majority of which are children under 5 years old [1]. The O-polysaccharide

\footnotetext{
* Correspondence: sunqiangzheng@icdc.cn

${ }^{\dagger}$ Equal contributors

${ }^{2}$ State Key Laboratory for Infectious Disease Prevention and Control,

Collaborative Innovation Center for Diagnosis and Treatment of Infectious

Diseases, National Institute for Communicable Disease Control and

Prevention, China CDC, P.O. Box 5, Changping, Beijing, China

Full list of author information is available at the end of the article
}

chain of the lipopolysaccharide (LPS) called O-antigen is an important and highly variable cell component presented on the outer leaflet of the outer membrane. It provides the basis for serotyping of S. flexneri, and plays an important role in the pathogenesis of shigellosis $[2,3]$. The immune response against the $\mathrm{O}$-antigen can induce protection that makes it a promising candidate as a component of shigellosis vaccines [4-7].

Based on the composition and structures of the Oantigens, $S$. flexneri can be divided into two main groups: one for serotypes $1-5, \mathrm{X}, \mathrm{Y}$, and 7 , and the other for serotype 6. Members of the former group share a linear O- 
polysaccharide backbone composed of tetrasaccharide repeats (O-units) of one $N$-acetylglucosamine (GlcNAc) and three L-rhamnose residues $\left.\left(\mathrm{Rha}^{\mathrm{I}}-\mathrm{Rha}^{\mathrm{III}}\right): \rightarrow 2\right)-\alpha-\mathrm{L}$ Rha $p^{\mathrm{III}}-(1 \rightarrow 2)-\alpha-\mathrm{L}-\mathrm{Rha}^{\mathrm{II}}-(1 \rightarrow 3)-\alpha-\mathrm{L}-\mathrm{Rha} p^{\mathrm{I}}-(1 \rightarrow 3)-\beta-$ $\mathrm{D}-\mathrm{Gl} p \mathrm{NAc}-(1 \rightarrow[8,9]$. Adding various chemical groups (glucosyl, acetyl or/and phosphoethanolamine) to different sugars of the tetrasaccharide backbone gives rise to diverse $\mathrm{O}$-antigen structures and, correspondingly, to various serotypes $[10,11]$. Serotype 6 has a different linear Opolysaccharide backbone with a tetrasaccharide repeat containing one residue each of $\mathrm{N}$-acetylgalactosamine (GalNAc) and galacturonic acid (GalA) and two rhamnose residues $\left.\left(\mathrm{Rha}^{\mathrm{II}}-\mathrm{Rha}^{\mathrm{III}}\right): \rightarrow 2\right)-\alpha-\mathrm{L}-\mathrm{Rhap}^{\mathrm{III}}-(1 \rightarrow 2)$ $\alpha-\mathrm{L}-\mathrm{Rha} p^{\mathrm{II}}-(1 \rightarrow 4)-\beta-\mathrm{D}-\mathrm{Gal} p \mathrm{~A}-(1 \rightarrow 3)-\beta-\mathrm{D}-\mathrm{Gal} p \mathrm{NAc}-(1 \rightarrow$ [12]. The $\rightarrow 2)-\alpha-\mathrm{L}-\mathrm{Rha}^{\mathrm{III}}-(1 \rightarrow 2)-\alpha-\mathrm{L}-\mathrm{Rha} p^{\mathrm{II}}$ disaccharide fragment is common for the $\mathrm{O}$-polysaccharides of both groups of S. flexneri. The observed O-antigen distinction reflects the genetic diversity of $S$. flexneri and different evolutionary origins of serotype 6 compared to other serotypes, which belong to different lineages of Shigella clones of Escherichia coli [13,14].

Similar to other Shigella species, the O-antigen gene cluster involved in the biosynthesis of the S. flexneri Opolysaccharide backbone is located between the housekeeping genes galF and gnd on the chromosome [15]. It contains three main classes of genes: (i) genes of nucleotide sugar biosynthesis pathways; (ii) glycosyltransferase genes; and (iii) $\mathrm{O}$-unit processing genes including those for O-antigen flippase $(w z x)$ and $\mathrm{O}$-antigen polymerase (wzy). Serotypes 1-5, X, Y, and 7 have similar O-antigen gene clusters whereas in serotype 6 the gene cluster is different, resulting in the two dissimilar O-antigen backbone structures of $S$. flexneri [15]. The factors responsible for modification of the backbone including $\mathrm{O}$-antigen glucosylation ( $\mathrm{tr}$ cluster), O-acetylation (oac and $o a c B$ ), and phosphorylation (opt) are carried by prophages, transposon-like structures, or plasmids outside of the Oantigen gene cluster $[10,11,16,17]$.

Modification of the $\mathrm{O}$ antigen by $\mathrm{O}$-acetylation has been also found in the serotype 6 . It was first reported at position 3 of Rha ${ }^{\mathrm{III}}$ [12], and later found that $\mathrm{Rha}^{\mathrm{III}}$ is partially 3-O-acetylated (major type) and partially 4-Oacetylated (minor type) [18]. The degree of 3/4-O-acetylation varies between strains and is thought to contribute to the serospecificity, which gives rise to a serological distinction between strains with lower ( 30\% and 15\%) and higher ( $\sim 60 \%$ and $30 \%)$ degrees of O-acetylation at position 3 and 4 (serotypes 6 and $6 a$, respectively) [18].

A similar 3/4-O-acetylation on Rha ${ }^{\mathrm{III}}$ also occurs in serotypes $1 \mathrm{a}, 1 \mathrm{~b}, 2 \mathrm{a}, 5 \mathrm{a}$, and Y of S. flexneri $[16,18,19]$, and the $\mathrm{O}$-acyltransferase-encoding gene, named $o a c B$, has recently been found to be responsible for this modification in these serotypes [16]. $o a c B$ is carried by a transposonlike structure located upstream of the $a d r A$ gene on the chromosome [16]. Further studies have indicated that the 3/4-O-acetylation on $\mathrm{Rha}^{\mathrm{III}}$ is widespread in serotypes $1 \mathrm{a}$, $1 \mathrm{~b}, 2 \mathrm{a}, 5 \mathrm{a}, 6$, and $\mathrm{Y}$ and confers to the bacterium a novel antigenic determinant provisionally named group $\mathrm{O}$-factor 9 [20]. However, the $o a c B$ gene cannot be detected from serotype 6 strains carrying 3/4-O-acetylation [16], indicating that another unknown gene is involved in the $\mathrm{O}$ antigen modification in this serotype.

Considering that the $\mathrm{O}$-antigen plays a key role in the serospecificity and virulence of Shigella, elucidation of $\mathrm{O}$-antigen modification mechanisms is important for understanding the $\mathrm{O}$-antigen biosynthesis, antigenicity, and pathogenicity of S. flexneri, as well as for shigellosis vaccine development. In this study, we identified the acyltransferase gene, named oacC, which mediates the 3/4-O-acetylation on $\mathrm{Rha}^{\mathrm{III}}$ in serotype 6 . The oacC gene is located in a phage-like structure on the chromosome and has an interchangeable function with the $o a c B$ gene of serotypes $1 \mathrm{a}, 1 \mathrm{~b}, 2 \mathrm{a}, 5 \mathrm{a}$, and Y. However, PCR screening indicated that gene oacC is specific to serotype 6, whereas $a a c B$ is specific to the other serotypes of $S$. flexneri.

\section{Results and Discussion}

Identification of an 0-acyltransferase gene, oacC, on the chromosome of S. flexneri serotype 6

Although the O-antigen of $S$. flexneri serotype 6 carries $3 / 4-\mathrm{O}$-acetylation on $\mathrm{Rha}^{\mathrm{III}}[12,16,18]$, the oacB gene responsible for the same modification in serotypes $1 \mathrm{a}, 1 \mathrm{~b}$, $2 \mathrm{a}, 5 \mathrm{a}$, and $\mathrm{Y}$ could not be detected from serotype 6 strains [16]. To identify potential factors associated with $3 / 4-\mathrm{O}$-acetylation of serotype 6 , we used the OacB protein sequence of serotype 2a strain Sf301 (Accession No. NP_706267.1) to search against the GenBank protein database. A predicted acyltransferase protein encoded by gene SGF_00264 of S. flexneri strains CDC 796-83 and SFCCH060_3012 of CCH060 was found to show the highest homology (72\% identity) to OacB (Figure 1). BLAST search revealed that these strains both carry within their genomes the serotype $6 \mathrm{O}$-antigen-specific gene $w z x$ [21], indicating that they belong to serotype 6. Data presented below suggest that this $\mathrm{OacB}$ homolog is responsible for the 3/4-O-acetylation on $\mathrm{Rha}^{\mathrm{III}}$ in serotype 6; hence it was named $\mathrm{OacC}$ and the encoding gene $o a c C$, following the designations for $\mathrm{OacB}$ and $o a c B$ [16].

OacC possesses conserved domains of the acyltransferase family (COG1835 or acyl_trans_3). It showed 28-39\% identity to predicted acyltransferases of Pseudomonas sp., Dechloromonas aromatica, Flavobacterium columnare, and some other species. OacC was also compared to Oac (which we have suggested to rename OacA [16]), an Oacetyltransferase responsible for 2-O-acetylation on $\mathrm{Rha}^{\mathrm{I}}$ giving rise to group 6 antigenic determinant in $S$. flexneri serotypes $1 \mathrm{~b}, 3 \mathrm{a}, 3 \mathrm{~b}, 4 \mathrm{~b}$, and $7 \mathrm{~b}[8,9]$. The two proteins 


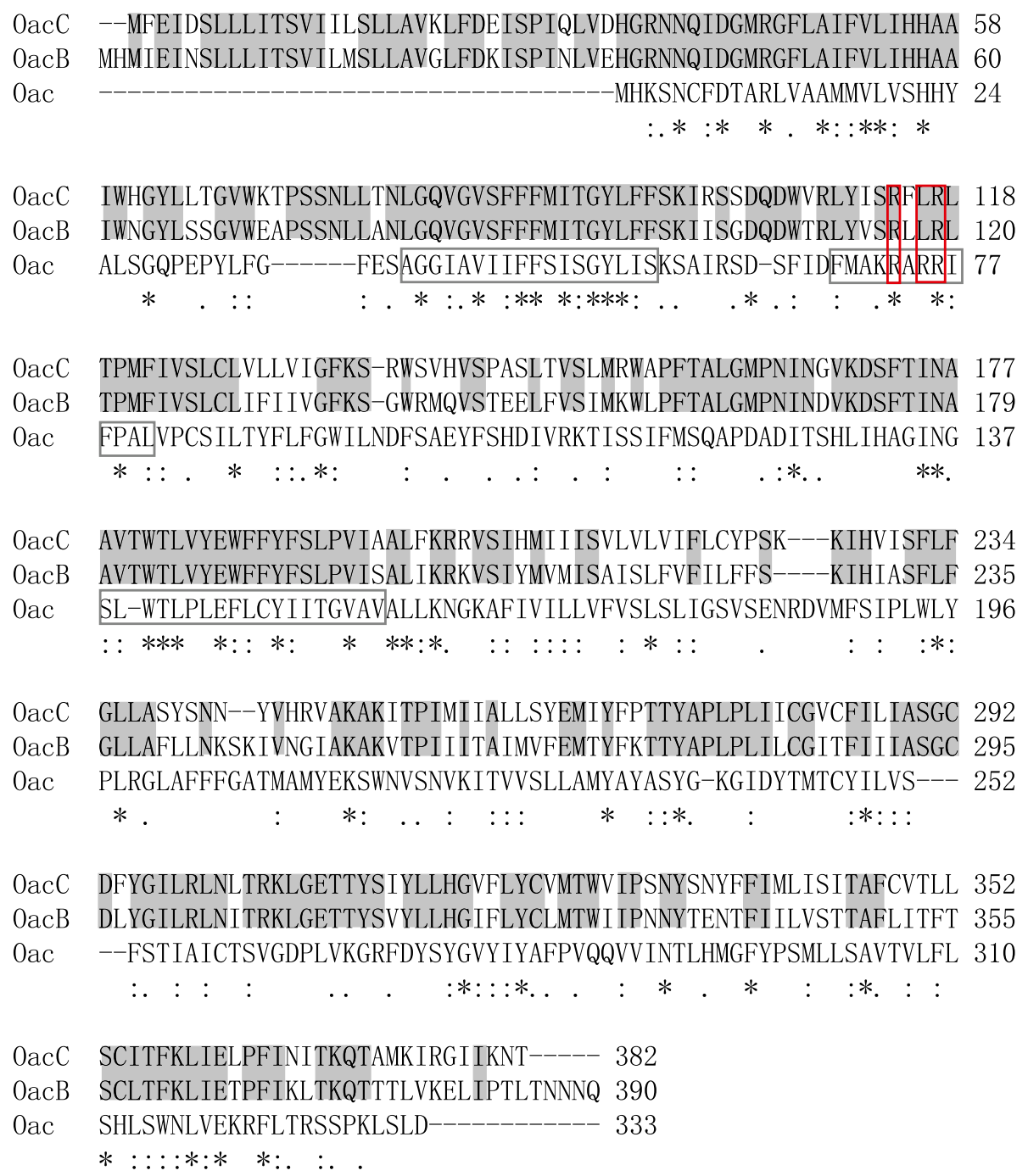

Figure 1 Sequence alignment of $\mathrm{OacB}, \mathrm{OacC}$, and $\mathrm{Oac}$ (or OacA), the proteins involved in O-acetylation modification of S. flexneri. Asterisks and dots indicate the amino acid residues that are identical or similar, respectively. Amino acids identical between OacB and OacC are shown in shadow. The three major regions conserved among the inner membrane trans-acylase family proteins are marked by black box. The three critical residues for the Oac (OacA) function are marked by red box.

presented higher homology in the three regions conserved among the inner membrane trans-acylase family proteins (amino acid residues 40 to 57, 69 to 81, and 138 to 156 of Oac) [22] (Figure 1). In particular, the residues R73 and R76, which are known to be critical for Oac functioning [23], are conserved in the three known acyltransferases (Figure 1).

To reveal a correlation between the presence of oacC and 3/4-O-acetylation in serotype 6, we performed PCR screening using the oacC-1 primer pair of serotype 6 strains G1671, G1038, and 51579 (Table 1), all of which are known to carry 3/4-O-acetylation on Rha ${ }^{\mathrm{III}}$. The expected PCR products (350-bp) were amplified from all three strains, and the PCR product sequences were fully identical to that of the SGF_00264 gene of CDC 796-83.
PCR screening of another 74 serotype 6 isolates collected in our laboratory showed that all isolates were PCR positive (Table 2). The 74 strains were also positive for O-factor 9 (Table 2) and therefore should carry 3/4-O-acetylation on Rha ${ }^{\text {III }}$. These results, combined with the functional analysis data described below, suggest that $\mathrm{OacC}$ is responsible for the 3/4-O-acetylation on Rha ${ }^{\text {III }}$ in serotype 6.

\section{Deletion and complementation analysis identifies the 3/4-O-acetylation function of oacC in serotype 6}

To confirm the function of the oacC gene, we performed oacC deletion and complementation assay on serotype 6 strain 51579 using the one-step inactivation of chromosomal genes method [26]. The kan-oacC primer pair (Table 3) carrying sequences complementary to $o a c C$ was 
Table 1 Strains and plasmids used in this study

\begin{tabular}{|c|c|c|}
\hline Strain or plasmid & Characteristic & $\begin{array}{l}\text { Reference } \\
\text { or source }\end{array}$ \\
\hline \multicolumn{3}{|l|}{ S. flexneri } \\
\hline 51579 & $\begin{array}{l}\text { Serotype } 6 \text {, carrying } 3 / 4-\mathrm{O}- \\
\text { acetylation on Rhall, used for } \\
\text { oacC gene cloning and inactivation } \\
\text { analysis, } \mathrm{Ap}^{\mathrm{s}}, \mathrm{Km}^{\mathrm{s}}\end{array}$ & [19] \\
\hline G1671, G1038 & $\begin{array}{l}\text { Serotype 6, carrying 3/4-O-acetylation } \\
\text { on Rha"l. }\end{array}$ & [18] \\
\hline $51579 \Delta$ oacC & $\begin{array}{l}\text { Strain } 51579 \text { with the oacC gene } \\
\text { replaced by the kanamycin resistance } \\
\text { gene (kan) from pSR551, } \mathrm{Km}^{\mathrm{r}}, \mathrm{Ap}^{\mathrm{s}}\end{array}$ & this study \\
\hline 51579 _oacC_pSQZ4 & $\begin{array}{l}51579 \Delta \text { oacC transformed by plasmid } \\
\text { pSQZ4 }\end{array}$ & this study \\
\hline 51579_oacC_pSQZ5 & $\begin{array}{l}\text { 51579 } \Delta \text { oacC transformed by plasmid } \\
\text { pSQZ5 }\end{array}$ & this study \\
\hline $\mathrm{Sf} 301 \Delta \mathrm{oac} B$ & $\begin{array}{l}\text { Strain } \mathrm{Sf} 301 \text { with the } \mathrm{oacB} \text { gene } \\
\text { inactive, } \mathrm{Km}^{\mathrm{r}}, \mathrm{Ap}^{\mathrm{s}}\end{array}$ & {$[16]$} \\
\hline Sf301_oacB_pSQZ4 & $\begin{array}{l}\text { Sf301 } \triangle \text { oacB transformed by plasmid } \\
\text { pSQZ4 }\end{array}$ & {$[16]$} \\
\hline Sf301_oacB_pSQZ5 & $\begin{array}{l}\text { Sf301 } \triangle \text { oacB transformed by plasmid } \\
\text { pSQZ5 }\end{array}$ & this study \\
\hline \multicolumn{3}{|l|}{ E. coli } \\
\hline $\mathrm{DH} 5 \mathrm{a}$ & $\begin{array}{l}\text { E. coli strain used for plasmid } \\
\text { propagation and gene cloning }\end{array}$ & TaKaRa \\
\hline \multicolumn{3}{|l|}{ Plasmid } \\
\hline pMD20T & T-A vector, $A p^{r}$ & TaKaRa \\
\hline pSR551 & $\mathrm{Km}^{\mathrm{r}}$, used for kan gene cloning & [24] \\
\hline pKOBEG & $\begin{array}{l}\text { A thermosensitive replicon that } \\
\text { carries the } \lambda \text { phage redy } \beta a \text { operon } \\
\text { expressed under the control of the } \\
\text { arabinose-inducible pBAD promoter }\end{array}$ & {$[25]$} \\
\hline pSQZ4 & $\begin{array}{l}\text { pMD20T carrying the whole sequence } \\
\text { of the } o a c B \text { gene from strain } S f 301, A p^{r}\end{array}$ & [16] \\
\hline pSQZ5 & $\begin{array}{l}\text { pMD20T carrying the whole sequence } \\
\text { of the oacC gene from strain 51579, } \\
\text { Ap }^{r}\end{array}$ & This study \\
\hline
\end{tabular}

employed to amplify the aminoglycoside 3'-phosphotransferase encoding gene $\left(\mathrm{Km}^{\mathrm{r}}\right)$ from plasmid pRS551. The PCR amplicon (831-bp) transferred into strain 51579 would recombine with the $\mathrm{oacC}$ gene resulting in a part of the oacC gene sequence (599-bp, 217 to 815 base) being replaced by the $\mathrm{Km}^{\mathrm{r}}$ gene. The deletion mutant $51579 \Delta$ oacC was selected on chloramphenicol and kanamycincontaining plate and detected by PCR amplification of the oacC gene using the oacC-1 and oacC-2 primer pairs (Table 3). The product of 350-bp which was amplified from the wild-type strain, was not obtained from the mutant $51579 \Delta$ oacC using the oacC-1 primer pair. However, when the $o a c C-2$ primer pair was used, a PCR product of 1,681 bp was amplified from $51579 \triangle$ oacC, which, as expected, was longer than that from the wild-type strain 51579 (1,450-bp). The oacC deletion was further confirmed by
Table 2 PCR screening of oacC in various serotypes of S. flexneri

\begin{tabular}{|c|c|c|c|c|}
\hline Serotype & $\begin{array}{c}\text { Number } \\
\text { of strains } \\
\text { tested }\end{array}$ & $\begin{array}{c}\text { Number of } \\
\text { O-factor } 9 \\
\text { positive strains }\end{array}$ & $\begin{array}{c}\text { Number of } \\
\text { oacB PCR } \\
\text { positive strains }\end{array}$ & $\begin{array}{c}\text { Number of } \\
\text { oacC PCR } \\
\text { positive } \\
\text { strains } \\
\end{array}$ \\
\hline $1 a$ & 106 & 102 & 102 & 0 \\
\hline $1 b$ & 26 & 26 & 26 & 0 \\
\hline $1 c=7 a$ & 3 & 0 & 0 & 0 \\
\hline $1 d$ & 14 & 0 & 0 & 0 \\
\hline $2 a$ & 169 & 160 & 160 & 0 \\
\hline $2 b$ & 61 & 0 & 0 & 0 \\
\hline $3 a$ & 18 & 0 & 0 & 0 \\
\hline $3 b$ & 4 & 0 & 0 & 0 \\
\hline $4 a$ & 4 & 0 & 0 & 0 \\
\hline 4av & 4 & 0 & 0 & 0 \\
\hline $4 b$ & 4 & 0 & 0 & 0 \\
\hline $5 a$ & 14 & 9 & 9 & 0 \\
\hline $5 b$ & 5 & 0 & 0 & 0 \\
\hline$x$ & 50 & 0 & 0 & 0 \\
\hline$X v$ & 126 & 0 & 0 & 0 \\
\hline Y & 39 & 24 & 24 & 0 \\
\hline YV & 20 & 0 & 0 & 0 \\
\hline 6 & 77 & 77 & 0 & 77 \\
\hline $7 b$ & 4 & 0 & 0 & 0 \\
\hline
\end{tabular}

Table 3 Primers used in this study

\begin{tabular}{|c|c|c|c|}
\hline Primer & Primer sequence $\left(5^{\prime}-3^{\prime}\right)$ & Target gene & Reference \\
\hline \multirow[t]{2}{*}{ oacC-1 } & F: gtgacacagtaagagaggc & \multirow[t]{2}{*}{ oacC } & \multirow[t]{2}{*}{ NZ_AERO01000013 } \\
\hline & R: tggaagaaataatcagatag & & \\
\hline \multirow[t]{2}{*}{ oacc-2 } & $\begin{array}{l}\text { F: ccgacgttccattagcccaa } \\
\text { atctg }\end{array}$ & \multirow[t]{2}{*}{ oacC } & \multirow[t]{2}{*}{ NZ_AERO01000013 } \\
\hline & $\begin{array}{l}\text { R: gcttccctgttcatagtgga } \\
\text { acacc }\end{array}$ & & \\
\hline \multirow[t]{2}{*}{$\begin{array}{l}\text { kan- } \\
\text { oacC }\end{array}$} & $\begin{array}{l}\text { F: gccatcttcgtacttattcatca } \\
\text { tgccgctatttggcatggctactta } \\
\text { ttaaccggggtatggaaaactccg } \\
\text { cacgttgtgtctcaaaatct }\end{array}$ & \multirow[t]{2}{*}{ kan, oacC } & \multirow[t]{2}{*}{ NZ_AERO01000013 } \\
\hline & $\begin{array}{l}\text { R: cctgatgcgataagtataaag } \\
\text { caaacaccgcaaattatgagag } \\
\text { ggagtggagcgtagcgtcccg } \\
\text { tcaagtcagcgta }\end{array}$ & & \\
\hline \multirow[t]{2}{*}{ oacC-3 } & F: cccctgectctcttactgtg & \multirow{2}{*}{$\begin{array}{l}\text { oacC, } \\
\text { SGF_00268 }\end{array}$} & \multirow[t]{2}{*}{ NZ_AERO01000013 } \\
\hline & R: gaatatgctgcctgacctgt & & \\
\hline \multirow[t]{2}{*}{ oacC-4 } & $\begin{array}{l}\text { F: cagtaagagaggcagg } \\
\text { ggag }\end{array}$ & \multirow[t]{2}{*}{$\begin{array}{l}\text { oacC, } \\
\text { SFCCH060_3017 }\end{array}$} & \multirow[t]{2}{*}{ NZ_AKMW01000058 } \\
\hline & $\begin{array}{l}\text { R: gggcataagcagggcaa } \\
\text { gag }\end{array}$ & & \\
\hline
\end{tabular}


sequencing analysis of the 1,681-bp amplicon from the $51579 \Delta$ oac C mutants.

The serological features of the mutants were determined by an agglutination assay using Shigella antisera of Seiken and anti-O-factor 9 serum. The deletion of oacC did not affect the type VI antigenicity, with the $51579 \Delta$ oacC mutant presenting the same antiserum VI reactivity as the parental 51579 (Table 4). This observation is consistent with and confirm the results of Hygge Blakeman et al. [27], who found that E. coli O147 (which possesses the same structure as that of serotype $6 \mathrm{O}$ antigen except that lacking $\mathrm{O}$-acetylation on $\mathrm{Rha}^{\mathrm{III}}$ ) present the same monoclonal antibody MASF VI-1 reactivity. In contrast, compared to the wild type, the mutant lost the reactivity with 3/4-O-acetylated $\mathrm{Rha}^{\mathrm{III}}$ specific anti-O-factor 9 serum (Table 4). The O-factor 9 antigenicity of the $51579 \mathrm{\Delta oacC}$ mutant was restored by complementation with a functional oacC-carrying plasmid pSQZ5 (Table 4).

The O-factor 9 antigenicity was further confirmed by an immunoblotting assay. The LPSs samples were resolved on 15\% SDS-PAGE gel and visualized by silver-staining (Figure 2A). A typical ladder-like banding pattern of an LPS with an $\mathrm{O}$-antigen composed of various numbers of $\mathrm{O}$ units was observed for all strains with no obvious difference between the parental strain and oacC deletion and complementation mutants. In Western blot, anti-O-factor 9 serum recognized the ladder-like LPS bands of the functional oacC-carrying strains (51579 wild type and 51579 oacC_pSQZ5 complementation mutant) but not the $51579 \Delta$ oacC deletion mutant (Figure 2B).

These findings were confirmed by structure analysis using ${ }^{1} \mathrm{H}$ and ${ }^{13} \mathrm{C}$ NMR spectroscopy of the Opolysaccharides isolated from the LPSs. In the spectra of the wild-type 51579 O-polysaccharide, there were signals for the 3-O-acetyl group (major) and 4-O-acetyl group (minor) at $\delta_{\mathrm{H}} 2.14$ and $2.19, \delta_{\mathrm{C}} 21.9$ and 21.8 , respectively. Due to electron deshielding effects of $\mathrm{O}$ -

\section{Table 4 Serotyping of wild-type strains, oacC and oacB deletion mutants, and complementation transformants by plasmid PSQZ4 or PSQZ5}

\begin{tabular}{|c|c|c|c|c|c|c|c|c|c|c|}
\hline \multirow[t]{2}{*}{ Strains } & \multicolumn{10}{|c|}{ Reactivity with typing and grouping antisera } \\
\hline & I & II & III & IV & V & VI & 3,4 & 6 & 7,8 & 9 \\
\hline 51579 & - & - & - & - & - & + & - & - & - & + \\
\hline $51579 \Delta$ oacC & - & - & - & - & - & + & - & - & - & \\
\hline 51579 & - & - & - & - & - & + & - & - & - & + \\
\hline $51579 \Delta$ oacC_pSQZ5 & - & - & - & - & - & + & - & - & - & + \\
\hline Sf301 & - & + & - & - & - & - & + & - & - & + \\
\hline $\mathrm{Sf301 \Delta oacB} \mathrm{[16]}$ & - & + & - & - & - & - & + & - & - & - \\
\hline Sf301 & - & + & - & - & - & - & + & - & - & + \\
\hline Sf301_oacB_pSQZ5 & - & + & - & - & - & - & + & - & - & + \\
\hline
\end{tabular}

acetylation [28], parts of the signals for $\mathrm{H}-3 / \mathrm{C}-3$ and $\mathrm{H}-$ $4 / \mathrm{C}-4$ of $\mathrm{Rha}^{\mathrm{III}}$ were shifted downfield to $\delta_{\mathrm{H}} / \delta_{\mathrm{C}} 5.04 /$ 74.2 and $4.80 / 75.7$ as compared with their positions in the non-O-acetylated $\mathrm{Rha}^{\mathrm{III}}$ at $\delta_{\mathrm{H}} / \delta_{\mathrm{C}} 3.83 / 71.2$ and $3.38 / 73.6$, respectively (compare published data for serotype 6a strain G1671 [18]). The degrees of Oacetylation determined by relative intensities of the ${ }^{1} \mathrm{H}$ NMR signals for various $\mathrm{O}$-acetylated and non-Oacetylated Rha ${ }^{\mathrm{III}}$ forms were $\sim 50 \%$ at position 3 and $\sim 30 \%$ at position 4. The ${ }^{1} \mathrm{H}$ and ${ }^{13} \mathrm{C}$ NMR spectra of the $51579 \Delta$ oacC mutant O-polysaccharide lacked any signals for $\mathrm{O}$-acetyl groups, and the positions of resonances of $\mathrm{H}$ $3 / \mathrm{C}-3$ and $\mathrm{H}-4 / \mathrm{C}-4$ of Rha ${ }^{\mathrm{III}}$ at $\delta_{\mathrm{H}} / \delta_{\mathrm{C}} 3.84 / 71.2$ and 3.36/ 73.7, respectively, indicated that they did not undergo any deshielding. The spectra of the 51579 $\Delta$ oacC_pSQZ5 transformant were essentially identical to those of the wild type. Therefore, the 51579 $\mathrm{OoacC}$ mutant lost the 3/4-O-acetylation on Rha ${ }^{\mathrm{III}}$, and this modification was restored by complementation of the mutant with a functional oacC gene (Figure 3).

It has been proposed that the degree of 3/4-O-acetylation on Rha ${ }^{\text {III }}$ contributes to the serospecificity of serotype 6 , which can be divided into subtypes 6 and $6 a$ with low and high degree of 3/4-O-acetylation, respectively [18]. We amplified and sequenced the $a a c C$ gene in serotype 6a strain G1671 and serotype 6 strain G1038 [18], and found no nucleotide difference between them. Hence, the variation in the degree of 3/4-O-acetylation is not due to sequence variation of the oacC gene, and most likely, results from different storage and/or cultivation conditions.

oacC of serotype 6 has the interchangeable 3/4-0acetylation function with oacB of serotypes $1 \mathrm{a}, 1 \mathrm{~b}, 2 \mathrm{a}, 5 \mathrm{a}$, and $Y$

To determine the distribution of oacC in S. flexneri, PCR screening of 671 strains of various non-6 serotypes (Table 2) was performed using the oacC-1 primer pair. No positive amplification was observed in any of the strains tested, including all $321 \mathrm{O}$-factor 9 positive strains of serotypes 1a, 1b, 2a, 5a, and Y, which do carry 3/4-O-acetylation (Table 2). We also PCR screened 12 strains of $S$. dysenteriae (one each of serotypes 1 to 12), 18 strains of $S$. boydii (one each of serotypes 1 to 18), 31 strains of $S$. sonnei and 10 strains of $E$. coli (one each of serogroups O6, O8, O13, O44, O71, O78, O127, O128, O157, O159) (Additional file 1: Table S1), and found that all were oacC negative. These data, combined with results of our previous studies on the $o a c B$ distribution [20], indicate that $o a c C$ is specific to serotype 6 , whereas $o a c B$ to the other serotypes of S. flexneri.

To elucidate whether the OacC protein has the interchangeable 3/4-O-acetylation function with $\mathrm{OacB}$, we transferred the oacC-carrying plasmid pSQZ5 into Sf301 $\triangle o a c B$ (serotype 2a strain Sf301 whose oacB gene is 


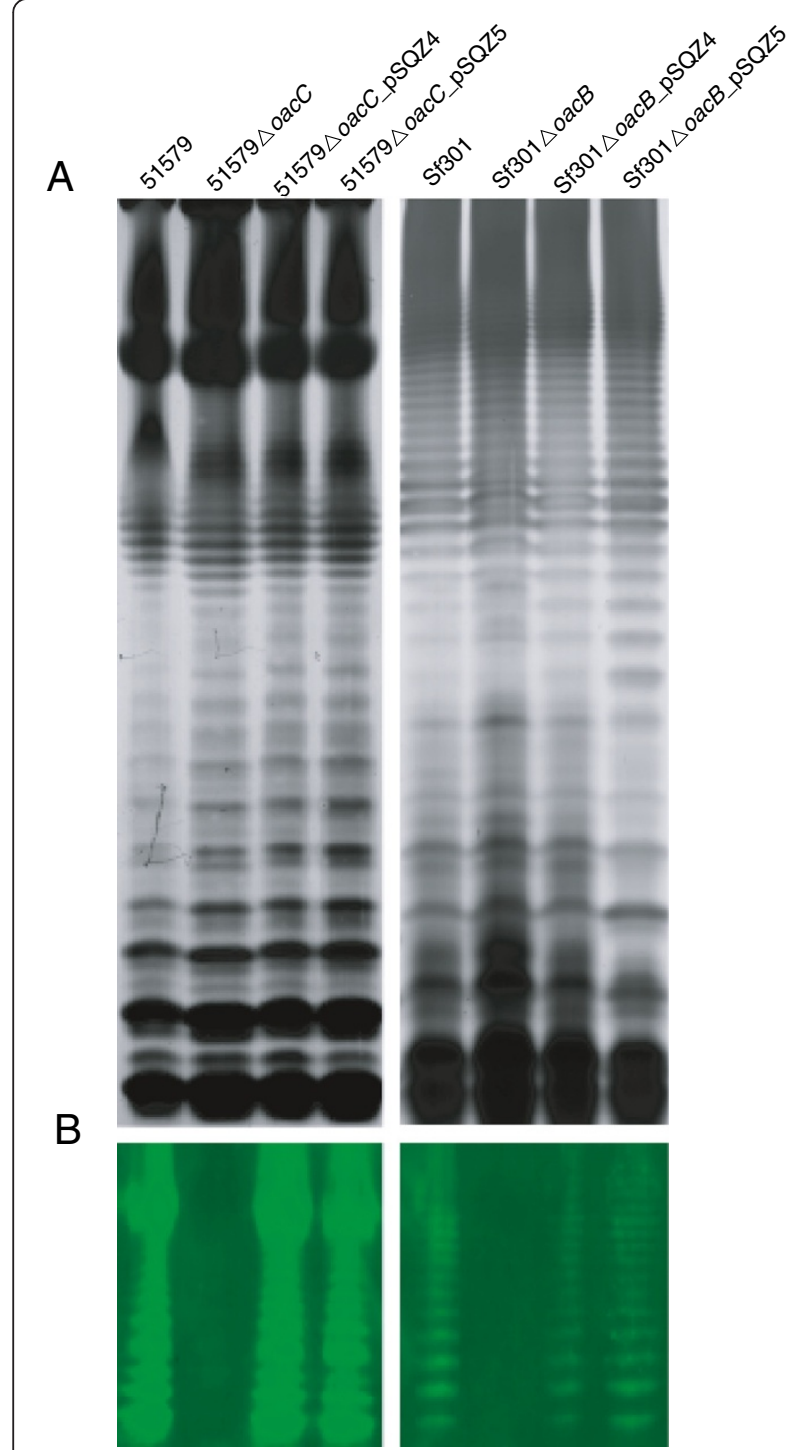

Figure 2 SDS-PAGE and Western blot of LPS from wild-type strains 51579 (serotype 6) and Sf301 (serotype 2a), their oacC and $O a c B$ deletion mutants, and complementation transformants by plasmids pSQZ4 and pSQZ5. A. Sliver-staining detection of LPS profiles on 15\% polyacrylamide gels. B. The LPS separated by SDS-PAGE were transferred onto a PVDF membrane and hybridized with anti-O-factor 9 serum. An anti-rabbit antibody labeled with fluorescent IRDye ${ }^{\mathrm{TM}} 800$ (Rockland) was used as the secondary antibody. Fluorescence was detected using Odyssey Infrared Imaging System (LI-COR).

inactivated) [16], and the oacB-carrying plasmid pSQZ4 [16] into 51579 $\mathrm{\Delta oacC}$, to construct complementation transformants Sf301 $\triangle o a c B_{-}$pSQZ5 and 51579 oacC $_{-}$ pSQZ4, respectively (Table 1). The serological features of the transformants were determined by an agglutination assay using Shigella antisera of Seiken (Danka Seiken, Japan) and anti-O-factor 9 serum, and compared to those

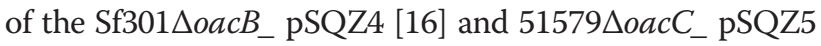
transformants (see above). It was found that both
Sf301 $\triangle o a c B_{-}$pSQZ5 and 51579 $\Delta$ oacC_ pSQZ4 acquired the agglutination reactivity with anti-O-factor 9 serum, and the serological features of the oacC transformants Sf301 $\triangle o a c B_{-}$pSQZ5 and 51579 $\triangle o a c C_{-}$pSQZ5 were same as those of the $o a c B$ transformants Sf301 $\triangle o a c B_{\text {_ pSQZ4 }}$ and $51579 \triangle$ oac $_{-}$pSQZ4, respectively (Table 4 ). In the immunoblotting assay, anti-O-factor 9 serum recognized the ladder-like LPS bands of Sf301 $\triangle o a c B_{-}$pSQZ4,

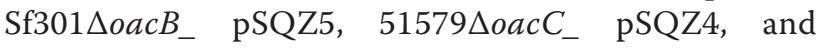
$51579 \triangle$ oac $_{-}$pSQZ5 with no obvious difference found between the $o a c B$ and $o a c C$ transformants in each host (Figure 2B).

The ability of $o a c B$ and $o a c C$ to transform the deletion mutants of the heterologous serotypes was further confirmed by ${ }^{1} \mathrm{H}$ and ${ }^{13} \mathrm{C}$ NMR spectroscopy, which showed that the O-polysaccharides of the transformants had acquired the 3/4-O-acetylation on Rha ${ }^{\text {III }}$ (Figure 3). The ${ }^{1} \mathrm{H}$ and ${ }^{13} \mathrm{C}$ NMR spectra of the O-polysaccharides of $51579 \triangle$ oac C _ pSQZ5 (characterized above in mutation and complementation analysis) and 51579 $\Delta$ oacC pSQZ4 were essentially identical, and, hence, the Opolysaccharides had the same structure, including the same total degree of 3/4-O-acetylation ( $80 \%$ in both). Serotype 2a strain Sf301, its $\mathrm{Sf} 301 \triangle$ oacB deletion mutant, and the Sf301 $\triangle o a c B_{-}$pSQZ4 transformant has been characterized by us earlier, and the ${ }^{1} \mathrm{H}$ and ${ }^{13} \mathrm{C}$ NMR chemical shifts of their O-polysaccharides have been reported [16]. The ${ }^{1} \mathrm{H}$ and ${ }^{13} \mathrm{C}$ NMR spectra of the Opolysaccharide of Sf301 $\triangle o a c B_{\text {_ }}$ pSQZ5 were similar to those of Sf301 $\triangle o a c B_{-}$pSQZ4, differing only in lower intensities in the former strain of the signals for the 3-Oacetyl and 4-O-acetyl groups at $\delta_{\mathrm{H}} 2.20$ and $2.14, \delta_{\mathrm{C}} 22.0$ and 22.1, as well as for the O-acetylated forms of Rha ${ }^{\mathrm{III}}$ : $\mathrm{H}-3 / \mathrm{C}-3$ at $\delta_{\mathrm{H}} / \delta_{\mathrm{C}} 5.09 / 74.1$ and $\mathrm{H}-4 / \mathrm{C}-4$ at $\delta_{\mathrm{H}} / \delta_{\mathrm{C}} 4.80 /$ 75.8. A lower degree of O-acetylation in the $\mathrm{Sf} 301 \triangle \mathrm{oacB}$ mutant complemented with the heterologous oac $C$ gene as compared with the homologous $a a c B$ gene (totally $~ 55 \%$ versus $~ 90 \%$ [16]) could be due to a poorer adaptation of OacC of serotype 6 for 3/4-O-acetylation of the serotype 2a O-polysaccharide having a different backbone structure.

\section{Gene oacC is carried by a bacteriophage structure on the} chromosome

The DNA regions flanking the oacC gene of partially sequenced serotype 6 strains CDC 796-83 and CCH060 were analyzed, and the genomic structures are shown in Figure 4. In strain CDC 796-83, the oac $C$ gene was located at the 5' end of contig NZ_AERO01000013 and immediately followed by 19 orfs encoding proteins of phage origin, with 15 of tail structure and assembly (SGF_00265- SGF_00279), two corresponding to headtail adaptor/connector (SGF_00281, SGF_00282), one putative phage protein (SGF_00283), and one related to head assembly (SGF_00284) (Figure 4, Additional file 2: 


$$
\begin{aligned}
& 51579 \\
& \text { 51579 } \triangle \text { oacC pSQZ4 } \\
& \text { 51579 } \text { oacC_pSQZ5 } \\
& \rightarrow 2)-\alpha-L-R h a p p^{\prime \prime \prime}-(1 \rightarrow 2)-\alpha-L-R h a p{ }^{\prime \prime}-(1 \rightarrow 3)-\beta-D-G a l p A-(1 \rightarrow 3)-\beta-D-G l c p N A c-(1 \rightarrow \\
& \text { | } 3 / 4 \\
& \text { OAc ( } 80 \%) \\
& 51579 \triangle \text { oacC } \\
& \rightarrow 2)-\alpha-L-R h a p{ }^{\prime \prime \prime}-(1 \rightarrow 2)-\alpha-L-R h a p{ }^{\prime \prime}-(1 \rightarrow 3)-\beta-D-G a l p A-(1 \rightarrow 3)-\beta-D-G l c p N A c-(1 \rightarrow \\
& \mathrm{S} f 301[16] \\
& \text { Sf301 } \triangle o a c B \_p S Q Z 4 \text { [16] } \\
& \rightarrow 2)-\alpha-\text { L-Rhap } p^{\prime \prime \prime}-(1 \rightarrow 2)-\alpha-\text {-Rhap }{ }^{\prime \prime}-(1 \rightarrow 3)-\alpha-L-R h a p^{\prime}-(1 \rightarrow 3)-\beta-D-G l c p N A c-(1 \rightarrow \\
& |3 / 4 \quad \uparrow 4 \quad| 6 \\
& \text { OAc ( 90\%) } \\
& \text { a-D-GIcp } \\
& \text { OAc }(\sim 60 \%) \\
& \mathrm{Sf} 301 \triangle \mathrm{oacB}[16] \\
& \rightarrow 2)-\alpha-L-R h a p p^{\prime \prime \prime}-(1 \rightarrow 2)-\alpha-L-R h a p p^{\prime \prime}-(1 \rightarrow 3)-\alpha-L-R h a p '-(1 \rightarrow 3)-\beta-D-G l c p N A c-(1 \rightarrow \\
& \uparrow 4 \quad \mid 6 \\
& \text { a-D-Glcp } \\
& \text { OAc }(\sim 60 \%) \\
& \text { Sf301 } \triangle 0 a c B \_p S Q Z 5 \\
& \rightarrow 2)-\alpha-L-R h a p{ }^{\prime \prime \prime}-(1 \rightarrow 2)-\alpha-L-R h a p \text { "I }-(1 \rightarrow 3)-\alpha-L-R h a p '-(1 \rightarrow 3)-\beta-D-G l c p N A c-(1 \rightarrow \\
& \text { | 3/4 } \\
& \text { OAc }(\sim 55 \%) \\
& \text { a-D-Glcp } \\
& 16 \\
& \text { OAc ( 50\%) }
\end{aligned}
$$

Figure 3 O-Polysaccharide structures of wild-type strains 51579 (serotype 6) and Sf301 (serotype 2a), their oacC and oacB deletion mutants, and complementation transformants by plasmids pSQZ4 and pSQZ5 [16].

Table S2). This prophage structure is homologous to ( $>95 \%$ identity at protein level), and organized in a similar manner as, the structure region (SfII_6-SfI__23) of S. flexneri bacteriophage SfII genome (NC_021857.1) (Figure 4), indicating their close evolutionary relationship. In contrast, it showed no similarity to the oac-carrying bacteriophage Sf6 and the oac1b-carrying phage-like structure, which are both responsible for the 2-O-acetylation on $\mathrm{Rha}^{\mathrm{I}}$ in serotypes $1 \mathrm{~b}, 3 \mathrm{a}, 3 \mathrm{~b}, 4 \mathrm{~b}$, and $7 \mathrm{~b}[17,29]$, or to the transposonlike structure carrying $o a c B$ for $3 / 4-\mathrm{O}$-acetylation of Rha ${ }^{\mathrm{III}}$ in serotypes $1 \mathrm{a}, 1 \mathrm{~b}, 2 \mathrm{a}, 5 \mathrm{a}$, and $\mathrm{Y}[16]$.

To elucidate whether the oacC-carrying prophage structure occurs in all serotype 6 strains, we performed PCR screening on 77 serotype 6 strains using the oac $C-3$ primer pair (Table 3), which covers the oacC gene and neighboring SGF_00268 genes. Except for 12 strains, the expected product (3,850-bp) could be amplified from all strains tested, and the PCR product sequences were identical to that of contig NZ_AERO01000013. The oacC gene of strain $\mathrm{CCH} 060$ was found to be located near the end of contig NZ_AKMW01000058 downstream of two insertion sequences (IS629 and IS630). They followed a region of genes encoding putative adhesin and attaching/effacing protein (SFCCH060_3018-SFCCH060_3019) (Figure 4), a fragment of unknown function that was also present in the genomes of several E. coli strains (ES11, LY180, $\mathrm{W}$, and KO11). The same insertion sequence IS630 also occurred immediately upstream of oacC in strain CDC 796-83 (Figure 4). Furthermore, PCR amplification and sequencing performed on the 77 serotype 6 strains using

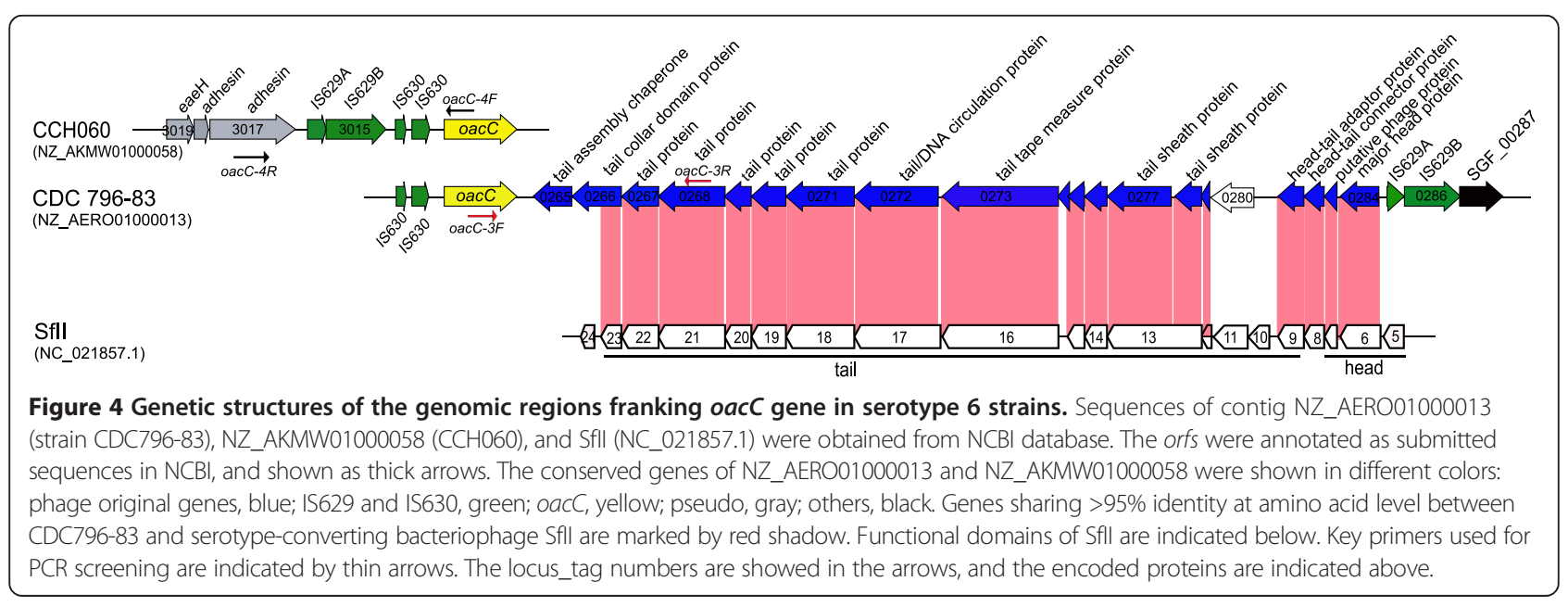


the oacC-4 primer pair that covers SFCCH060_3012 (oacC) and SFCCH060_3017, showed that all but 4 strains (HN157, HN12, G1038 and 51579) tested were PCR positive. The four outliers also were negative when further PCR amplification was performed targeting on genes up to SFCCH060_3019, probably owing to DNA fragment insertion or deletion events happened in this region. PCR screening using the oacC-3 primer pair indicated that the four strains carried the phage-like structure downstream of $\mathrm{oacC}$, and, therefore, might acquire the 3/4-O-acetylation by the same phage mechanism.

\section{Conclusions}

In this work, the oacC gene encoding a novel acyltransferase $\mathrm{OacC}$ was demonstrated to be responsible for the 3/4-O-acetylation on $\mathrm{Rha}^{\mathrm{III}}$ in S. flexneri serotype 6. This conclusion was supported by the following evidences: i) the OacC protein encoded by the oacC gene showed significant similarity to $\mathrm{OacB}$ and other acyltransferase family proteins; ii) deletion of the functional oacC gene resulted in the loss of the 3/4-O-acetylation in serotype 6; iii) the cloned oacC gene mediated the 3/4-O-acetylation of Rha ${ }^{\mathrm{III}}$ upon transformation. The oacC gene is specific to serotype 6 and presented in all strains of this serotype tested; hence, this gene can be used as a target for molecular identification of S. flexneri serotype 6 .

Although oac $C$ occurs only in serotype 6 strains, it has the interchangeable function with $\operatorname{oacB}$, which is responsible for 3/4-O-acetylation of Rha ${ }^{\mathrm{III}}$ in non-6 S. flexneri serotypes, and conversely $o a c B$ can confer this modification to serotype 6. This could be expected as oac $C$ and $o a c B$ possess a high homology (72\% identity), and the O-polysaccharides of serotype 6 and the other S. flexneri serotypes share the $\rightarrow 2)-\alpha-\mathrm{L}-\mathrm{Rha}^{\mathrm{III}}-(1 \rightarrow 2)-\alpha-\mathrm{L}-\mathrm{Rha} p^{\mathrm{II}}$ disaccharide fragment.

As opposite to the $o a c B$ gene localized in a transposonlike structure, the $o a c C$ gene is carried by a chromosomal phage-like structure adjacent to the adhesin region that is conserved in serotype 6. Therefore, the mechanisms of $3 /$ 4-O-acetylation in serotype 6 and the other serotypes $(1,2$, 5 and $\mathrm{Y}$ ) are distinct, which is not surprising as they have different evolutionary origins and belong to different lineages of Shigella clones of E. coli $[13,14]$. Accordingly, the divergent Oacs might have been gained from different species in independent events. These findings enhance our understanding of the genetic basis of $\mathrm{O}$-antigen modifications in S. flexneri.

\section{Methods}

\section{Bacterial strains, plasmids, and culturing conditions}

Strains and plasmids used in this study are listed in Table 1. S. flexneri serotype 6 strain 51579 [16] carrying 3/4-O-acetylation on $\mathrm{Rha}^{\mathrm{III}}$ in the $\mathrm{O}$-antigen was used as the reference strain for oacC gene cloning and deletion analysis. The oacC gene deletion mutant 51579 $\Delta$ oacC and $o a c B$ gene deletion mutant Sf301 $\Delta o a c B$ [16] were employed as hosts for the plasmid pSQZ4 and pSQZ5 transformation analysis. Seventy-seven S. flexneri serotype 6 strains, 671 strains of other serotypes of S. flexneri (Table 2), 12 strains of $S$. dysenteriae (one each of serotypes 1 to 12 ), 18 strains of $S$. boydii (one each of serotypes 1 to 18 ), 31 strains of $S$. sonnei and 10 strains of E. coli (one each of serogroups O6, O8, O13, O42, O71, O78, O127, O128, O157, O159) were used for oacC gene PCR detection analysis (Additional file 1: Table S1). S. flexneri strains were either isolates from patients in a surveillance program performed by China CDC during 2000 to 2012 or were purchased from the National Collection of Type Cultures (NCTC) or were kindly donated by B. Liu (Nankai University, Tianjin). E. coli DH5 $\alpha$ was used for plasmid propagation. pMD20T vector (TaKaRa, Japan) was used for DNA sequencing and oacC gene function analysis. Plasmid pRS551 was used for kanamycin resistance gene amplification. pKOBEG encoding a homologous recombination system was used in oacC gene deletion analysis. Strains were grown in a $37^{\circ} \mathrm{C}$ incubator or orbital shaker in Luria-Bertani (LB) medium supplemented with ampicillin $\left(100 \mu \mathrm{g} \mathrm{ml}^{-1}\right)$, kanamycin $\left(40 \mu \mathrm{g} \mathrm{ml}^{-1}\right)$, or chloramphenicol $\left(50 \mu \mathrm{g} \mathrm{ml}^{-1}\right)$ when appropriate.

\section{Bioinformatics analysis}

The protein sequence of $\mathrm{O}$-acyltransferase for 3/4-Oacetylation (OacB) (accession No. NP_706267.1) of S. flexneri strain Sf301 (serotype 2a) was searched against the GenBank protein database, using the BLASTP web server (http://www.ncbi.nlm.nih.gov/BLAST). Homologs of OacB were aligned using the ClustalW2 program in the EMBLEBI (http://www.ebi.ac.uk/Tools/msa/clustalw2/).

\section{DNA techniques}

Primer pairs used in this study are listed in Table 3. The oac $C-1$ primer pair was used for oacC gene detection. The oacC-2 primer pair was used for oacC gene function analysis. The $o a c C-3$ and $o a c C-4$ primer pairs were used to amplify regions up and downstream of oacC in serotype 6 isolates. Oligonucleotide primers were synthesized by Sangon Biotech (Shanghai). PCR amplifications were performed using a TaKaRa PCR Amplification Kit (Takara, Japan) following a standard protocol. PCR products amplified from strain 51579 using the oacC-2 primer pair were purified and cloned into the T-vector pMD20T (TaKaRa, Japan), which carries an additional $\mathrm{T}$ at both 3' terminus and can complement the A base of the PCR product, to generate the pSQZ5 expression plasmid. The recombinant plasmids were first transformed into commercial $E$. coli DH5 $\alpha$ competent cells (TaKaRa, Japan), and then into $S$. flexneri strains tested, using a standard protocol [30]. The 
transformants were selected on LB plates supplemented with ampicillin $\left(100 \mu \mathrm{g} \mathrm{ml}^{-1}\right)$ and further confirmed by PCR amplification of the oacC gene.

\section{oacC gene functional deletion and complementation analysis}

Deletion of the $\mathrm{oacC}$ gene was performed on S. flexneri serotype 6 strain 51579 using a one-step method as described previously [26]. The kanamycin resistance gene $\left(\mathrm{Km}^{\mathrm{r}}\right)$ was PCR amplified from plasmid pRS551 using the kan-oacC primer pair (Table 3). The PCR products were electroporated into strain 51579 carrying plasmid pKOBEG (encoding a homologous recombination system for oacC gene inactivation) and selected on an LB plate with chloramphenicol $\left(50 \mu \mathrm{g} \mathrm{ml}^{-1}\right)$ and kanamycin $\left(40 \mu \mathrm{g} \mathrm{ml}^{-1}\right)$. oacC gene deletion mutant $51579 \Delta$ oacC was confirmed by a slide agglutination assay using antiO-factor 9 serum [20] and PCR amplification of oacC using the oacC-1 and oacC-2 primer pairs. Plasmid pSQZ5 and pSQZ4 [16] were transferred into $51579 \triangle$ oacC and Sf301 $\triangle o a c B$ [16], giving rise to complemented strains

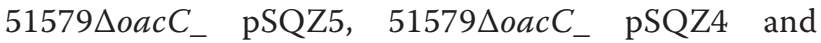
Sf301 $\triangle$ oacB_pSQZ5, respectively.

\section{Serotyping analysis}

The serological features of S. flexneri strains were determined by a slide agglutination test using commercially available Shigella monovalent antisera kit (Denka Seiken, Japan) and 3/4-O-acetylated Rha ${ }^{\mathrm{III}}$-specific anti-O-factor 9 serum prepared previously [20].

\section{Western blot assay}

LPSs were prepared using an LPS extraction Kit (iNtRON, South Korea) according to the manufacturer's instructions. The LPSs were electrophoresed on $15 \%$ polyacrylamide gels and detected by silver staining as described [31]. A Western blot assay of the LPSs was performed as described [20]. Briefly, the LPSs separated by SDS-PAGE were transferred onto a polyvinylidene difluoride (PVDF) and incubated with anti-O-factor 9 serum. After washing, the membrane was incubated with anti-rabbit antibody labeled with fluorescent IRDye 800 (Rockland). The fluorescence was detected using an Odyssey infrared imaging system (LI-COR).

\section{O-polysaccharide isolation and structure analysis}

For O-polysaccharide structure analysis, the LPSs of wild-type strains, deletion mutants and transformants were isolated by phenol-water extraction of bacterial cells [32]. The crude extract without separation of layers was dialyzed against tap water, nucleic acids and proteins were precipitated by adding aqueous $50 \% \mathrm{CCl}_{3} \mathrm{CO}_{2} \mathrm{H}$ at $4^{\circ} \mathrm{C}$ to reach $\mathrm{pH} 2$, the supernatant was dialyzed against distilled water and freeze-dried. The purified LPSs obtained in yields of $5 \%$ to $7 \%$ were hydrolyzed with aqueous $2 \%$ acetic acid at $100^{\circ} \mathrm{C}$ until formation of a lipid precipitate (1-2 h), and the released O-polysaccharides were isolated in yields of $12 \%$ to $34 \%$ from the supernatant by gel permeation chromatography on Sephadex G-50 Superfine (Amersham Biosciences, Sweden) in $0.05 \mathrm{M}$ pyridinium acetate buffer ( $\mathrm{pH} 4.5)$ monitored with a differential refractometer (Knauer, Germany).

Structures of the O-polysaccharides were elucidated using two-dimensional nuclear magnetic resonance (NMR) spectroscopy, including a ${ }^{1} \mathrm{H},{ }^{13} \mathrm{C}$ heteronuclear single-quantum coherence (HSQC) experiment, essentially as described previously [33]. Positions of O-acetyl groups were determined by characteristic low-field displacements of NMR signals for ${ }^{1} \mathrm{H}$ and

${ }^{13} \mathrm{C}$ atoms at the $\mathrm{O}$-acetylation sites. The degree of $\mathrm{O}$ acetylation was determined by relative integral intensities of the ${ }^{1} \mathrm{H}$ NMR signals for the $\mathrm{O}$ - and $\mathrm{N}$-acetyl groups, 3-O- and 4-O-acetylated and non-O-acetylated Rha ${ }^{\text {III }}$.

\section{Availability of supporting data}

All supporting data are available and included as additional files (Additional file 1: Table S1 and Additional file 2: Table S2).

\section{Additional files}

Additional file 1: Table S1. Shigella and E. coli strains used for the oacC gene PCR detection and anti-O-factor 9 serum specificity evaluation.

Additional file 2: Table S2. Analysis of predicted ORFs of oacC-carrying contig NZ_AERO01000013 of serotype 6 strain CDC 796-83.

\section{Competing interests}

The authors declare that they have no competing interests. As the bacterial isolates included in the study had no patient information, ethical approval and appropriate consent from patients were unnecessary.

\section{Authors' contributions}

QS and YAK designed the study and co-drafted the manuscript. SNS, AMS, and ASS performed the O-antigen isolation and structure analysis. JW carried out the PCR amplification and DNA sequencing. PD and NZ participated in the bioinformatics analysis. XL carried out the oacC gene one-step deletion assay. RL and JX participated in the manuscript drafting. All authors read and approved the final manuscript.

\section{Acknowledgements}

YAK, SNS, AMS, and ASS were supported by the Russian Science Foundation (14-14-01042); JW, XL, RL, PD, NZ, JX, and QS were supported by the National Natural Science Foundation of China (No. 81271788); the National Basic Research Priorities Program (2011CB504901); the Project of State Key Laboratory for Infectious Disease Prevention and Control (2011SKLID203); the National Key Program for Infectious Diseases of China (2013ZX10004221, 2013ZX10004216-001-002, 2012ZX10004215).

\section{Author details}

${ }^{1}$ N.D. Zelinsky Institute of Organic Chemistry, Russian Academy of Sciences, Moscow, Russian Federation. ${ }^{2}$ State Key Laboratory for Infectious Disease Prevention and Control, Collaborative Innovation Center for Diagnosis and Treatment of Infectious Diseases, National Institute for Communicable Disease Control and Prevention, China CDC, P.O. Box 5, Changping, Beijing, 
China. ${ }^{3}$ School of Biotechnology and Biomolecular Sciences, University of New South Wales, Sydney NSW 2052, Australia.

Received: 30 June 2014 Accepted: 9 October 2014

Published online: 21 October 2014

\section{References}

1. Bardhan P, Faruque AS, Naheed A, Sack DA: Decrease in shigellosis-related deaths without Shigella spp.-specific interventions, Asia. Emerg Infect Dis 2010, 16(11):1718-1723.

2. Morona R, Daniels C, Van Den Bosch L: Genetic modulation of Shigella flexneri 2a lipopolysaccharide $O$ antigen modal chain length reveals that it has been optimized for virulence. Microbiology 2003, 149(Pt 4):925-939.

3. West NP, Sansonetti P, Mounier J, Exley RM, Parsot C, Guadagnini S, Prevost MC, Prochnicka-Chalufour A, Delepierre M, Tanguy M, Tang CM: Optimization of virulence functions through glucosylation of Shigella LPS. Science 2005, 307(5713):1313-1317.

4. Phalipon A, Tanguy M, Grandjean C, Guerreiro C, Belot F, Cohen D, Sansonetti PJ, Mulard LA: A synthetic carbohydrate-protein conjugate vaccine candidate against Shigella flexneri 2a infection. J Immunol 2009, 182(4):2241-2247.

5. Robbins JB, Kubler-Kielb J, Vinogradov E, Mocca C, Pozsgay V, Shiloach J, Schneerson R: Synthesis, characterization, and immunogenicity in mice of Shigella sonnei O-specific oligosaccharide-core-protein conjugates. Proc Natl Acad Sci U S A 2009, 106(19):7974-7978.

6. Kubler-Kielb J, Vinogradov E, Mocca C, Pozsgay V, Coxon B, Robbins JB, Schneerson R: Immunochemical studies of Shigella flexneri 2a and 6, and Shigella dysenteriae type 10 -specific polysaccharide-core fragments and their protein conjugates as vaccine candidates. Carbohydr Res 2010, 345(11):1600-1608.

7. Passwell JH, Ashkenzi S, Banet-Levi Y, Ramon-Saraf R, Farzam N, Lerner-Geva L, Even-Nir H, Yerushalmi B, Chu C, Shiloach J, Robbins JB, Schneerson R, The Israeli Shigella Study Group: Age-related efficacy of Shigella O-specific polysaccharide conjugates in 1-4-year-old Israeli children. Vaccine 2010, 28(10):2231-2235.

8. Kenne L, Lindberg B, Petersson K, Katzenellenbogen E, Romanowska E: Structural studies of Shigella flexneri O-antigens. Eur J Biochem 1978, 91(1):279-284

9. Foster RA, Carlin NI, Majcher M, Tabor H, Ng LK, Widmalm G: Structural elucidation of the O-antigen of the Shigella flexneri provisional serotype 88-893: structural and serological similarities with S. flexneri provisional serotype Y394 (1c). Carbohydr Res 2011, 346(6):872-876.

10. Allison GE, Verma NK: Serotype-converting bacteriophages and O-antigen modification in Shigella flexneri. Trends Microbiol 2000, 8(1):17-23.

11. Sun Q, Knirel YA, Lan R, Wang J, Senchenkova SN, Jin D, Shashkov AS, Xia S, Perepelov AV, Chen Q, Wang Y, Wang H, Xu J: A novel plasmid-encoded serotype conversion mechanism through addition of phosphoethanolamine to the O-antigen of Shigella flexneri. PLoS One 2012, 7(9):e46095.

12. Dmitriev BA, Knirel YA, Sheremet OK, Shashkov AA, Kochetkov NK, Hofman IL: Somatic antigens of Shigella. The structure of the specific polysaccharide of Shigella newcastle (Sh. flexneri type 6) lipopolysaccharide. Eur J Biochem 1979, 98(1):309-316

13. Pupo GM, Lan R, Reeves PR: Multiple independent origins of Shigella clones of Escherichia coli and convergent evolution of many of their characteristics. Proc Natl Acad Sci U S A 2000, 97(19):10567-10572.

14. Pupo GM, Karaolis DK, Lan R, Reeves PR: Evolutionary relationships among pathogenic and nonpathogenic Escherichia coli strains inferred from multilocus enzyme electrophoresis and mdh sequence studies. Infect Immun 1997, 65(7):2685-2692.

15. Liu B, Knirel YA, Feng L, Perepelov AV, Senchenkova SN, Wang Q, Reeves PR, Wang L: Structure and genetics of Shigella $O$ antigens. FEMS Microbiol Rev 2008, 32(4):627-653

16. Wang J, Knirel YA, Lan R, Senchenkova SN, Luo X, Perepelov AV, Wang Y, Shashkov AS, Xu J, Sun Q: Identification of an O-acyltransferase gene (oacB) that mediates 3- and 4-O-acetylation of rhamnose III in Shigella flexneri $O$ antigens. J Bacteriol 2014, 196(8):1525-1531.

17. Sun $Q$, Lan R, Wang Y, Wang J, Xia S, Wang Y, Zhang J, Yu D, Li Z, Jing H, $\mathrm{Xu} \mathrm{J}$ : Identification of a divergent O-acetyltransferase gene oac $1 b$ from Shigella flexneri serotype 1b strains. Emerg Microbes Infect 2012, 1(9):e21.

18. Perepelov AV, Shekht ME, Liu B, Shevelev SD, Ledov VA, Senchenkova SN, LVov VL, Shashkov AS, Feng L, Aparin PG, Wang L, Knirel YA: Shigella flexneri O-antigens revisited: final elucidation of the O-acetylation profiles and a survey of the $\mathrm{O}$-antigen structure diversity. FEMS Immunol Med Microbiol 2012, 66(2):201-210.

19. Sun Q, Knirel YA, Lan R, Wang J, Senchenkova SN, Shashkov AS, Wang Y, Wang Y, Luo X, Xu J: Dissemination and serotype modification potential of PSFxv_2, an O-antigen PEtN modification plasmid in Shigella flexneri. Glycobiology 2014, 24(3):305-313.

20. Wang J, Lan R, Knirel YA, Luo X, Senchenkova SN, Shashkov AS, Xu J, Sun Q: Serological identification and prevalence of a novel O-antigen epitope linked to 3- and 4-O-acetylated rhamnose III of lipopolysaccharide in Shigella flexneri. J Clin Microbiol 2014, 52(6):2033-2038.

21. Cheah KC, Beger DW, Manning PA: Molecular cloning and genetic analysis of the rfb region from Shigella flexneri type 6 in Escherichia coli K-12. FEMS Microbiol Lett 1991, 67(2):213-218.

22. Slauch JM, Lee AA, Mahan MJ, Mekalanos JJ: Molecular characterization of the oafA locus responsible for acetylation of Salmonella typhimurium $O$-antigen: oafA is a member of a family of integral membrane trans-acylases. J Bacteriol 1996, 178(20):5904-5909.

23. Thanweer F, Tahiliani V, Korres H, Verma NK: Topology and identification of critical residues of the O-acetyltransferase of serotype-converting bacteriophage, SF6, of Shigella flexneri. Biochem Biophys Res Commun 2008, 375(4):581-585.

24. Simons RW, Houman F, Kleckner N: Improved single and multicopy lac-based cloning vectors for protein and operon fusions. Gene 1987, 53(1):85-96.

25. Pradel N, Ye C, Livrelli V, Xu J, Joly B, Wu LF: Contribution of the twin arginine translocation system to the virulence of enterohemorrhagic Escherichia coli 0157:H7. Infect Immun 2003, 71(9):4908-4916.

26. Datsenko KA, Wanner BL: One-step inactivation of chromosomal genes in Escherichia coli K-12 using PCR products. Proc Natl Acad Sci U S A 2000, 97(12):6640-6645.

27. Hygge Blakeman K, Weintraub A, Widmalm G: Structural determination of the O-antigenic polysaccharide from the enterotoxigenic Escherichia coli 0147. Eur J Biochem 1998, 251(1-2):534-537.

28. Jansson PE, Kenne L, Schweda E: Nuclear magnetic resonance and conformational studies on monoacetylated methyl D-gluco-and D-galacto-pyranosides. J Chem Soc Perkin Trans 1987, 1(2):377-383.

29. Casjens S, Winn-Stapley DA, Gilcrease EB, Morona R, Kuhlewein C, Chua JE, Manning PA, Inwood W, Clark AJ: The chromosome of Shigella flexneri bacteriophage Sf6: complete nucleotide sequence, genetic mosaicism, and DNA packaging. J Mol Biol 2004, 339(2):379-394.

30. Sambrook J, Fritsch EF: T. Maniatis: Molecular cloning: a laboratory manual. New York: Cold Spring Harbor Laboratory Press; 1989.

31. Morona R, Brown MH, Yeadon J, Heuzenroeder MW, Manning PA: Effect of lipopolysaccharide core synthesis mutations on the production of Vibrio cholerae O-antigen in Escherichia coli K-12. FEMS Microbiol Lett 1991, 66(3):279-285.

32. Westphal O, Jann K: Bacterial lipopolysaccharides. Extraction with phenol-water and further applications of the procedure. Meth Carbohydr Chem 1965, 5:83-90.

33. Knirel YA, Lan R, Senchenkova SN, Wang J, Shashkov AS, Wang Y, Perepelov $A V$, Xiong $Y, X U$ J, Sun Q: O-antigen structure of Shigella flexneri serotype $\mathrm{Yv}$ and effect of the lpt-O gene variation on phosphoethanolamine modification of S. flexneri O-antigens. Glycobiology 2013, 23(4):475-485.

doi:10.1186/s12866-014-0266-7

Cite this article as: Knirel et al:: Genetic and structural identification of an O-acyltransferase gene (oacC) responsible for the 3/4-O-acetylation on rhamnose III in Shigella flexneri serotype 6. BMC Microbiology 2014 14:266 